\title{
Applications of Computer Simulation in Mechanism Teaching
}

\author{
TSENG-TI FU \\ Department of Mechanical Engineering, National Taiwan University, Taipei, Taiwan 10660, Republic of China
}

Received 26 March 2002; accepted 28 May 2003

\begin{abstract}
The scope of implementing commercially available dynamics software as an aid to mechanism teaching and research in the university is reviewed. Based on the computer simulation, it has been applied to demonstrations in mechanism teaching, system learning and validation, and performance predictions. They are found useful in raising students' interests by vivid graphics, and impressing them with almost the same effect as dealing with real mechanisms. Despite the complexity of numerical schemes, the effort would be paid off by the flexibility and economy in modeling various systems with fewer limitations. The applications can be easily adapted to the specific needs of learning and in future network education. ๑ 2003 Wiley Periodicals, Inc. Comput Appl Eng Educ 11: 156-165, 2003; Published online in Wiley InterScience (www.interscience.wiley.com.); DOI 10.1002/cae.10043
\end{abstract}

Keywords: computer simulation; mechanism teaching; engineering education

\section{INTRODUCTION}

Demonstration is one of the important tools in the field of engineering education. It helps students realize how theory and systems work in reality. This is particularly true for the teaching of design. Traditionally, physical models and workshop practice were applied for this purpose. However, either the preparation of physical models or practicing in the workshop is a time and resource consuming process for both

Correspondence to T.-T. Fu (ttfu@ccms.ntu.edu.tw) (C) 2003 Wiley Periodicals Inc. teaching and learning. Therefore, it would be beneficial if there is a way to reduce or replace the needs of making physical models in teaching engineering.

Owing to the development of computer technology, both in hardware and software, the speed and quality of computer simulation have been dramatically enhanced. Virtual simulation of physical systems in computer becomes feasible not only in terms of accuracy but also friendliness in its interfaces. Graphics and modularized elements have made computer simulation an illustrative task. Many numerical codes are available in the market [1], such as ADAMS, DADS, and AUTOSIM, etc. The process of teaching demonstrations can then be virtualized. 
Costs, resources, and time spent in the traditional physical production are greatly reduced.

On the other hand, as engineering systems become more complicated, students are required to learn more during the same period of time. Owing to the limits of school facilities, theories and physical phenomena are mainly delivered by illustrations and equations in classroom. Students essentially learn by graphs and their imagination. It is acknowledged that the best way to impress students and help them learn the theory is to see or practice how it works. In this regard, computer simulation can serve as an economical and effective alternative to acquire similar feelings as those from physical models. Students are able to deal with more realistic and complicated problems in school and within a shorter period of time.

By reviewing the experience and progress in mechanism teaching since the introduction of computer software in the department, it is hoped to shed light on its capability of teaching students more insights into engineering.

\section{DESCRIPTIONS OF THE COMPUTER SOFTWARE}

ADAMS is a commercially available software package for dynamic simulation. It acquires the name from the initials of "Automatic Dynamic Analysis of Mechanical Systems." This program is based on the principles of multi-body dynamics [2] for mechanism analysis. Simulating physical systems by solving the simultaneous equations of motion, software like ADAMS can normally be divided into three parts:

(1) Pre-processor: as a graphical interface for constructing the system elements into computer models [3].

(2) Processor: the numerical code of solving simultaneous dynamic equations of motion for the system [4].

(3) Post-processor: for the demonstration of analysis results, including system animation and various performance XY plots [3].

Fundamental knowledge of kinematics, dynamics, and numerical analysis is essential for the accurate modeling of physical systems and proper interpretations of simulation results.

Linking with other software packages, ADAMS is able to perform additional analyses in engineering. The connection with MATLAB allows ADAMS to incorporate various control algorithms into ordinary mechanical systems. Integration with the finite element program extends its scope into systems comprising flexible bodies.

\section{APPLICATIONS IN TEACHING ENVIRONMENTS}

To facilitate teaching and learning of the mechanism design for students in engineering, ADAMS has been applied to the following areas of study.

\section{Computer-Aided Teaching and Learning}

Owing to the nature of three dimensions, it is a common difficulty in a fundamental course like "Theory of Mechanisms" to demonstrate and explain the movement of spatial linkages in the classroom. In most cases, students can hardly imagine how links move and function as the lecturer taught. Occasions had occurred that students questioned about the mobility of a simple but typical RSSR spatial linkage as shown in Figure 1a [5]. Under a specific configuration that the axis of one fixed revolute pivot (e.g., the joint A in Fig. 1) pointed to the moving spherical joint on the other link (e.g., joint C), the Gruebler's equation of constraint [1] predicts its mobility as follows:

$$
M=6(4-1)-5 \cdot 2-3 \cdot 2=2
$$

Students find it difficult to comprehend how this particular linkage can move in space with two degrees of freedom. Especially, the linkage contends an idle degree of freedom which will not affect the joint angles of input and output links (links 2 and 4). A better way to convince them is to witness link movements by themselves. A physical teaching model can serve this purpose but may take time to build or need money to purchase. With animation in computer, it may only require a few hours to create a visual proof as shown in Figure 1b. Students can also easily distinguish the special type of motion by the idle degree of freedom from the demonstration of computer simulation.

Similar situations exist in other special linkages, such as the Oldham coupling in (Fig. 2a) [6]. With two rectangular bars perpendicular to each other and arranged on each side of the middle circular plate (part 3), students tend to think intuitively that the mechanism will get stuck in operation. Computer animation, as depicted in Figure 2b, helps them realize how relative movements take place between its 
components and have a better idea of the way this mechanism works.

Figure 3 is another example which is useful for students to understand the importance in choosing path curves for the calculations of relative acceleration between coincident points $\mathrm{P}_{2}$ and $\mathrm{P}_{3}$. According to the theory [5], calculations can be performed based on either link 2 or 3 as follows:

$$
\begin{aligned}
& \vec{A}_{P_{3}}=\vec{A}_{P_{2}}+\vec{A}_{P_{3} / P_{2}}^{n}+\vec{A}_{P_{3} / P_{2}}^{t}+2 \cdot \vec{\omega}_{2} \times \vec{v}_{P_{3} / P_{2}} \\
& \vec{A}_{P_{2}}=\vec{A}_{P_{3}}+\vec{A}_{P_{2} / P_{3}}^{n}+\vec{A}_{P_{2} / P_{3}}^{t}+2 \cdot \vec{\omega}_{3} \times \vec{v}_{P_{2} / P_{3}}
\end{aligned}
$$
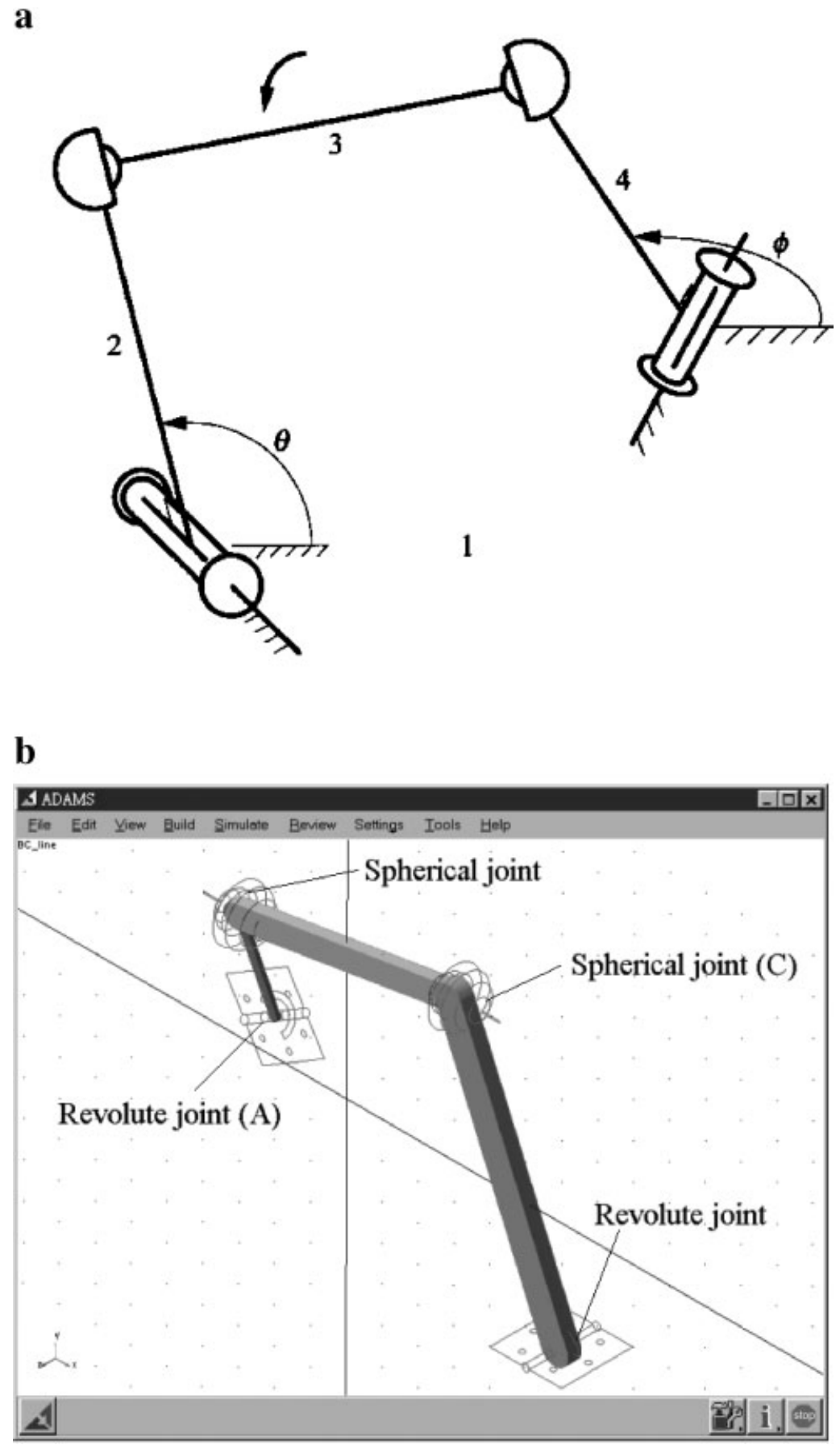

Figure 1 The RSSR spatial linkage. (a) The schematic physical model [5]. (b) The computer model. 
a
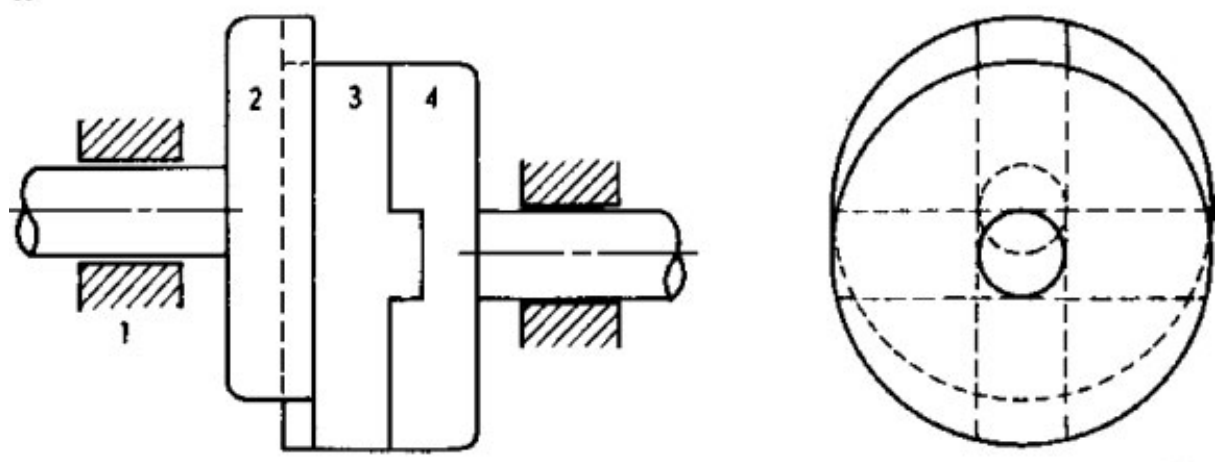

b

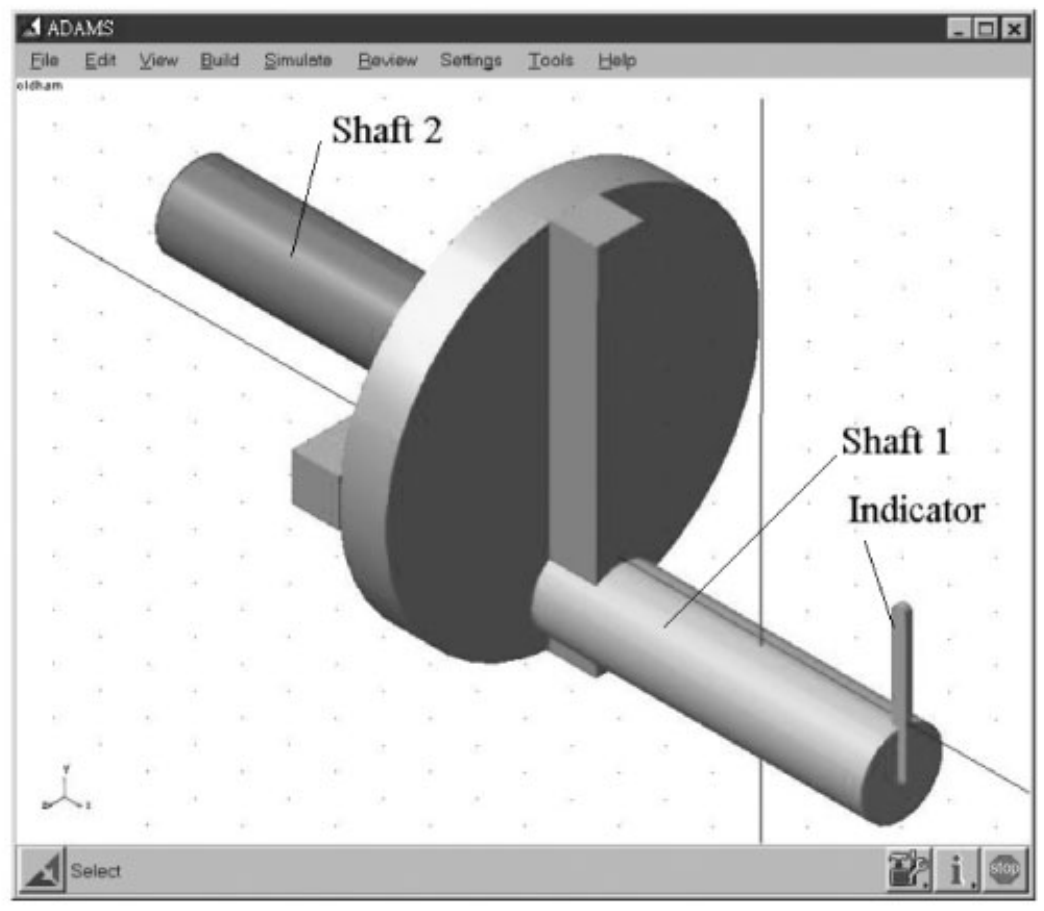

Figure 2 The Oldham coupling. (a) The schematic physical model [6]. (b) The computer model.

In which, $\vec{A}_{P_{2} / P_{3}}^{n}$ (or $\vec{A}_{P_{3} / P_{2}}^{n}$ ) is the component of relative acceleration between $\mathrm{P}_{2}$ and $\mathrm{P}_{3}$ along the common normal of their paths. Calculations of this component involves the radius of path curvature between $\mathrm{P}_{2}$ and $\mathrm{P}_{3}$. It becomes apparent from the simulation in Figure 3 that Equation 3 is a better choice for the calculations because of the simplicity in its associated path described on link 3 .

Feedback from students indicated that linkage motions demonstrated by computer did strongly impress them as a lively contrast to the theory being studied, and improve their overall understanding of mechanisms.
Being flexible and requiring fewer resources, it is easier for the teacher to respond specific requests from individual students. With basic skills of computer modeling, students can even verify their doubts or ideas by themselves. Simulation results could also be collected and put on the computer network to facilitate self-learning in relevant engineering subjects.

\section{Computer-Aided Validation}

ADAMS has been used to study the feasibility of ideas generated in practical design projects. Figure 4 shows 


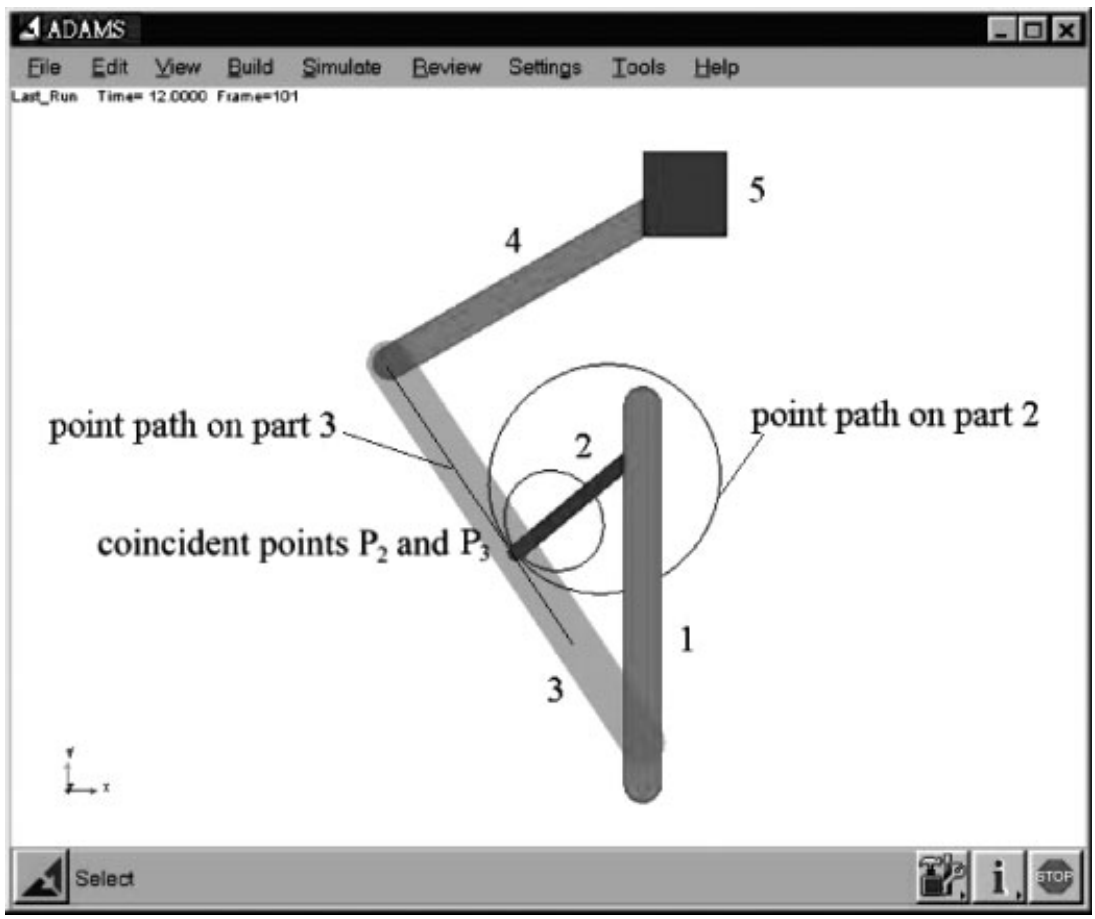

Figure 3 Path curves of coincident points of the quick return mechanism.

a planetary gear train designed for transmitting power of a direct-driven electric motor [7] for different operation modes in a new type of electric scooters. It was first calculated by theory and then validated in ADAMS dynamically and made predictions for its performance. Some mistakes were corrected in the process of simulation and chances of design changes in its physical production were reduced.

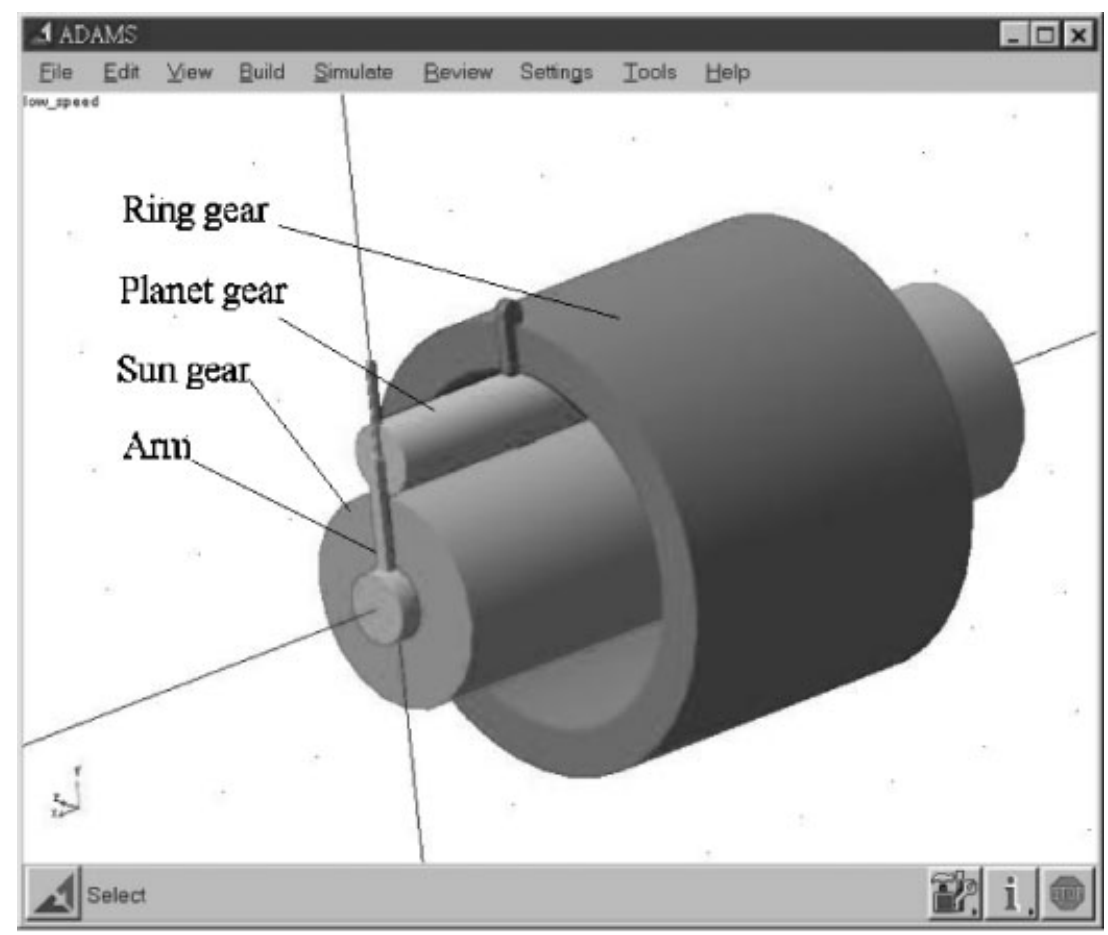

Figure 4 A planetary gear train model. 
By computer simulation, designs can be effectively validated without consuming materials and machining efforts in the workshop. Students are able to be more focused on improving their designs in the project and promptly acquiring the feedback from computer.

Projects for mechanism analysis by computer software are also established for undergraduates. In these projects, students have to take basic training courses of ADAMS in the department, and are encouraged to apply their skills in investigating interesting mechanisms from their daily lives. By modeling system components in computer, they will have the opportunity to look into the mechanism in great details for its parts, constraints, and force interactions. Upon completion of the model, motion of this mechanism is simulated. In this way, students are able to learn more of the mechanism in question to suit their specific interests and depth than in a general teaching class.

Under this scheme, mechanisms been attempted by now include automotive steering system [8], suspension, tire-road interactions, scooter, escalator, the escapement mechanism of clock, tray motion of CD-ROM, windshield wiper, centrifugal clutch, Geneva wheel, and even snake movements, etc. Figures 5, 6, and 7 are samples of students' work from these projects.

In particular, while modeling the centrifugal clutch (Fig. 7), students managed to conduct an ex- periment corresponding to the simulation conditions and investigate their difference [9]. Figure 8 shows results from both approaches for the drum rotational speed against that of the driving plate due to the action of this clutch. According to the students participated in this project, the contrast in this figure stimulated their interest in deeper thinking of system behaviors. In the course of pursuing reasons for the discrepancy, they gained a further insight into the significance of design factors on system performance, and acknowledged the role of computer analysis in modern engineering.

\section{Computer-Aided Research}

The ability of predicting system performance by computer is also helpful in research, especially for the study of complicated systems or new technology which has little prior experience. For research involving practical applications, complicated systems governed by a large amount of equations are normally inevitable. With adequate data inputs, computer models could be a satisfactory substitute for analysis. The need of formulating dynamic equations of motion and following calculations is replaced by building graphical models and constraints in computer. By saving the work of deriving equations, it frees researcher's mind with more time in generating new ideas and investigating physical meanings of results. The only thing to be cautioned is that validation by

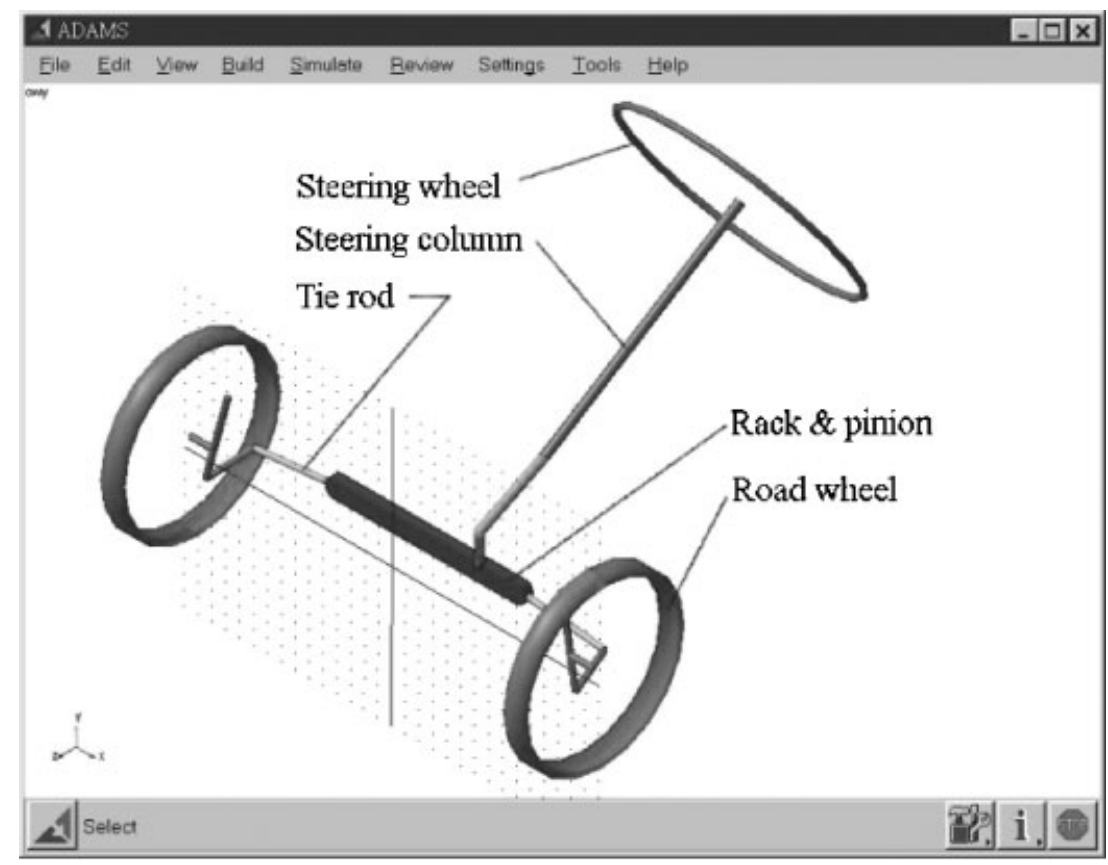

Figure 5 An automotive steering model. 


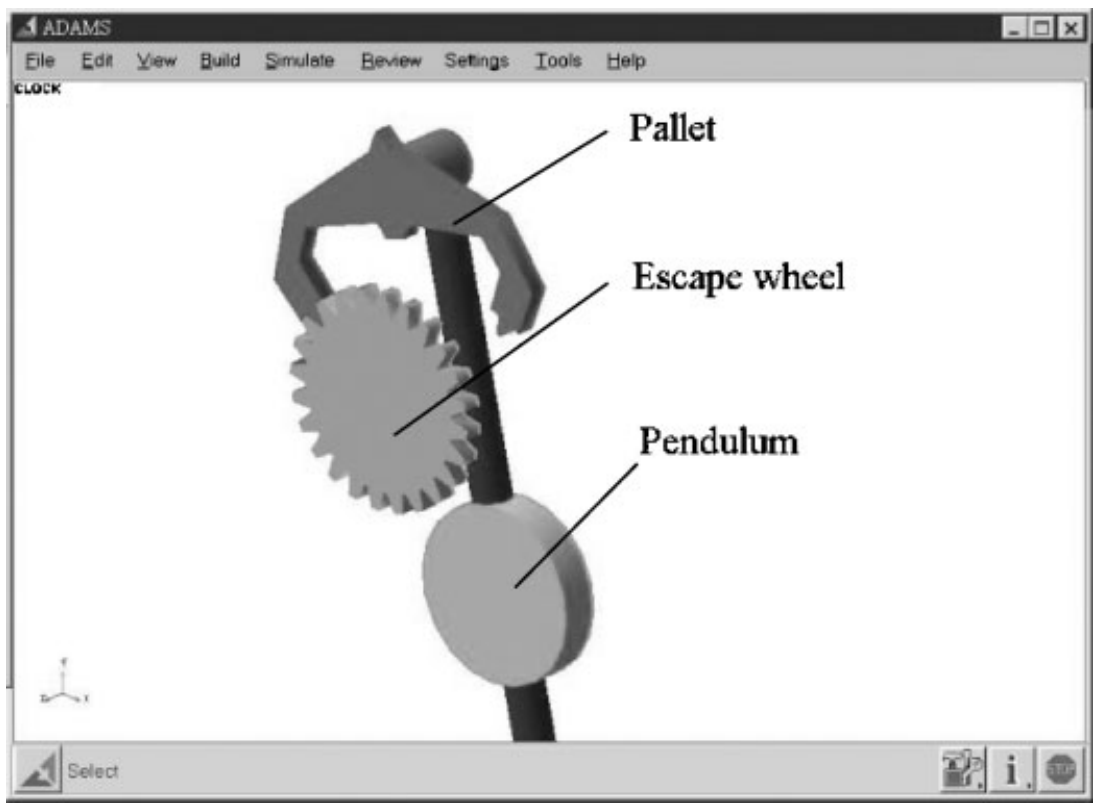

Figure 6 A model for the escapement mechanism of clock.

some fundamental tests to ensure the accuracy of input data and computer model is always indispensable.

Another useful application in research is the evaluation of different types of systems with essentially the same functions. In reality, these systems may exist only in different models and comparisons will not be fair if they were evaluated under different conditions. Only with the same settings can the true strengths and weaknesses be faithfully revealed.

Taking research into the quantitative evaluation of automotive steering systems as an example [10],

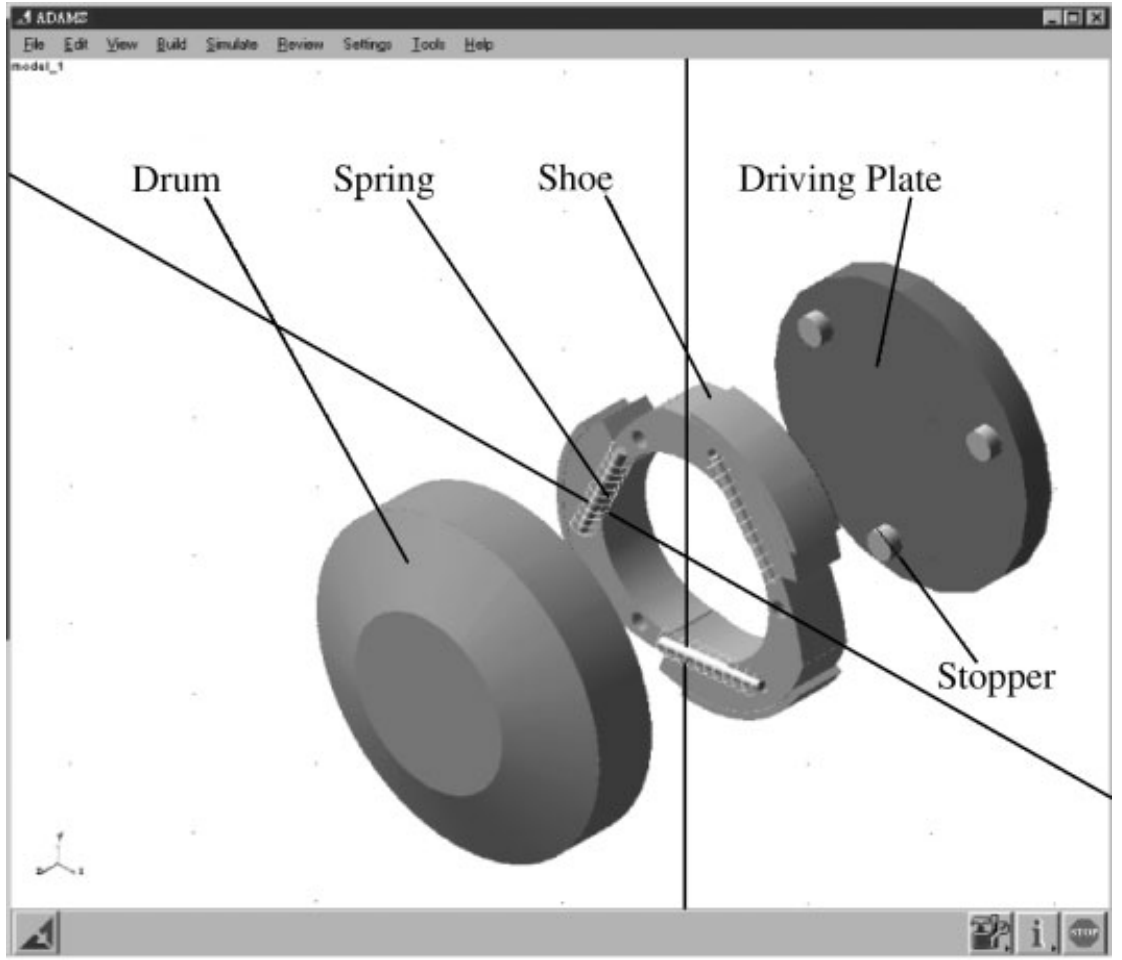

Figure 7 A centrifugal clutch model. 


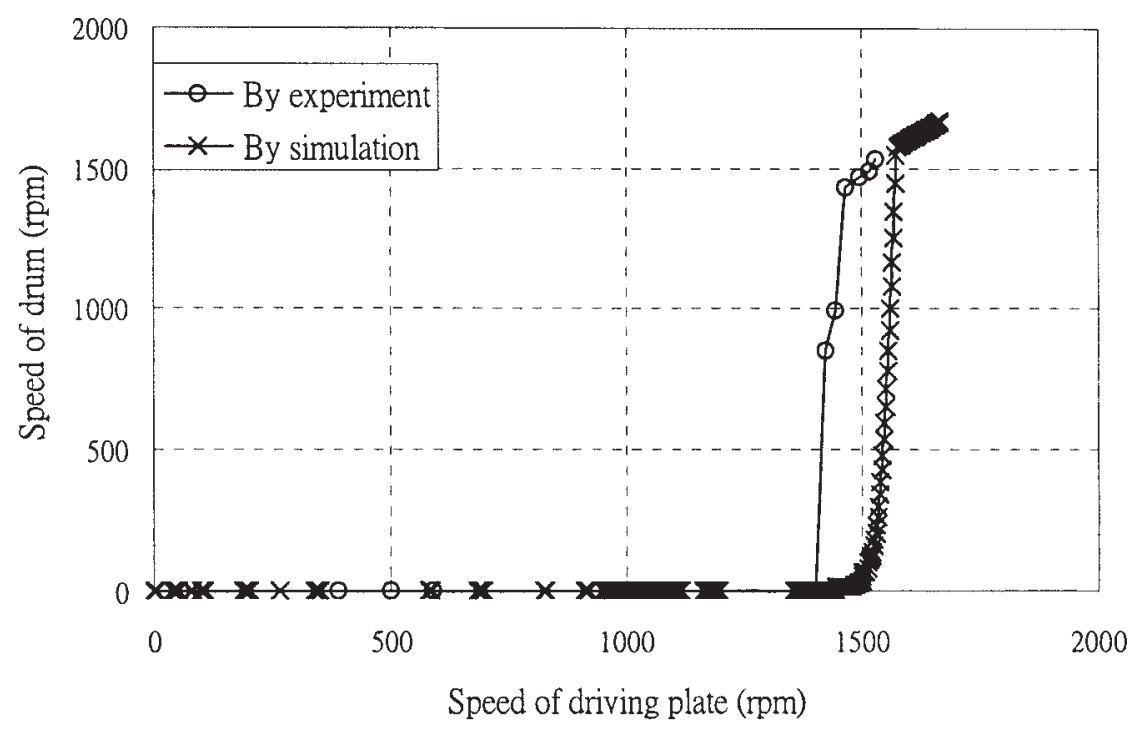

Figure 8 Comparisons of results from computer simulation and laboratory experiment.

there are currently four main types in the market, i.e., manual, hydraulic power assisted, electronically controlled hydraulic power assisted, and electric power assisted steering. As can be seen from the schematic diagrams in Figure 9 [11], they all share similar fundamental configurations to those of manual steering and only the other auxiliary parts distinguish their types. It is usually not possible for a car model to have versions equipped with all these steering types. An attempt to make comparisons in real world may result in much trouble and cost in producing necessary components. On the other hand, in virtual world, one can achieve this more easily by modifying components and parameters based on the same validated computer model.

\section{FINDINGS FROM THE APPLICATIONS}

From the analysis point of view, ADAMS is not the only choice to implement virtual simulation. Other commercially available software or even generalpurposed programs like FORTRAN, $\mathrm{C}++$, and MATLAB can all well perform the same task. It is the vivid graphics and friendly interfaces provide ADAMS alike with the ability of enhanced comprehension and easy implementation. They bring the users with almost the same feeling as dealing with physical objects.

Computer software can be an economical and efficient alternative to fulfill teaching purposes. Students are able to witness system movements without involving much factory work or purchasing teaching models. It is able to improve student's understanding and interest similar to those by physical models. In particular, physical models may be subject to wear out by the usage of demonstration and aging. For models in computer, less maintenance work is required.

To increase the efficiency of product development, engineers in industry have applied various software packages as an integrated tool in their design $(\mathrm{CAD})$, analysis (CAE), and manufacturing (CAM) tasks for a long time [12]. Incorporating computer software in teaching environments helps students be familiar with this important tool earlier and have a better connection with their future careers in the industry.

However, special care has to be taken when performing computer analysis. Incorrect results may arise from pitfalls in the numerical solving schemes. For example, the artificial damping originally included in a certain numerical integration algorithms to stabilize the iteration processes [2] may attenuate the amplitude of simulation results and make them deviate gradually from the way they should be in real situations. Users have to be prudent when applying these schemes and validate their results before they can actually trust what computer simulation predicts.

Accurate predictions of system behaviors by computer demand for correct data inputs and appropriate simulation skills. They are the key to the success of computer applications. Experience of applying computer software in mechanism teaching showed that some students who were attracted by vivid animation in the beginning might find difficulties 
$\mathbf{a}$

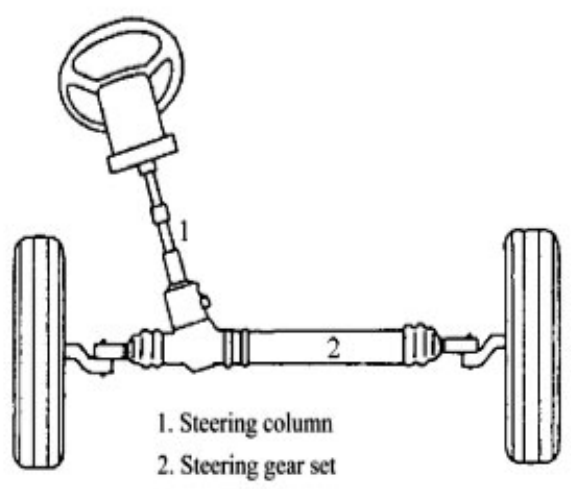

c

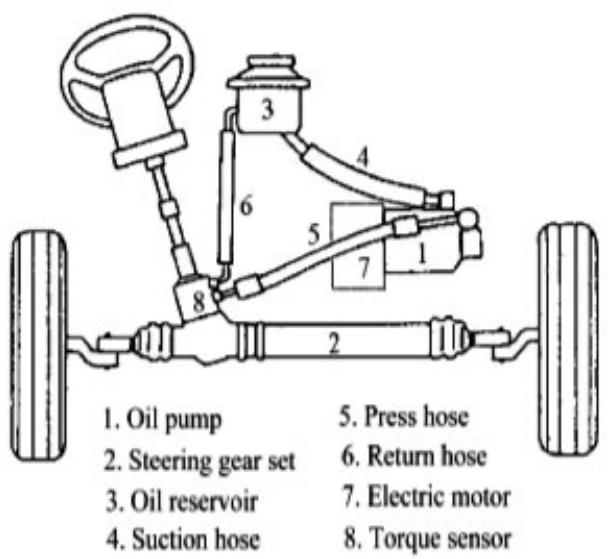

b

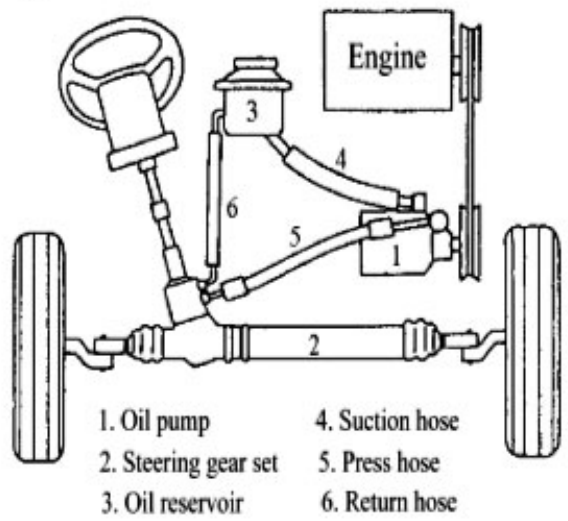

d

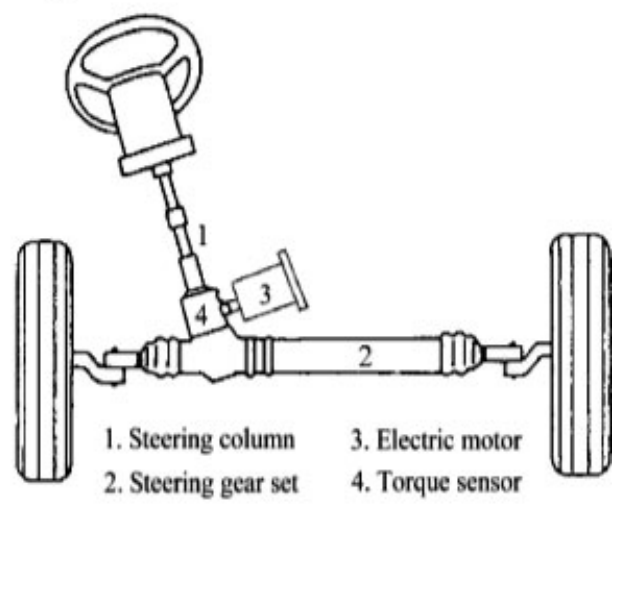

Figure 9 Schematic diagrams for four types of steering systems. (a) Manual steering. (b) Hydraulic power assisted steering [11]. (c) Electronically controlled hydraulic power assisted steering. (d) Electric power assisted steering.

when the computer model got involved. Encouraging students to work in groups to facilitate mutual discussions was found very helpful for teaching computer simulation especially in the early stage of learning.

\section{CONCLUSIONS}

Computer software has been applied in the aspects of teaching, learning, validation, and research in engineering education. It is found useful not only in industry but also in university. Computer simulation can efficiently raise student's interest of learning by vivid graphical construction and animation. This tool is flexible in creating computer models for various types of systems but requires skills to perform simulations properly.

Computer simulation is beneficial especially in terms of saving time and efforts for both teaching and learning in the education process. It can be applied continuously from the courses of fundamental subjects to advanced topics in engineering so that it covers the whole process of learning from undergraduate to postgraduate. Introduction of computer software in university also helps students have a good connection with their future work in industry.

Being less demanding in storage and preparations than traditional physical teaching models, computer simulation is a good integrated alternative for teaching demonstrations, self-learning aids, and computer network applications. 


\section{REFERENCES}

[1] A. G. Erdman and G. N. Sandor, Mechanism designAnalysis and synthesis, 3rd edn., Prentice-Hall International, Inc., 1997, pp 24-116.

[2] J. G. de Jalon and E. Bay, Kinematic and dynamic simulation of multibody systems: The real-time challenge, Springer-Verlag, 1994, p 245.

[3] Using ADAMS/View, User manual, Mechanical Dynamics, Inc., 1998.

[4] Using ADAMS/Solver, User manual, Mechanical Dynamics, Inc., 1998.

[5] K. J. Waldron and G. L. Kinzel, Kinematics, dynamics, and design of machinery, John Wiley \& Sons, Inc., 1999, pp 23-87.

[6] G. H. Martin, Kinematics and dynamics of machines, 2nd edn., McGraw-Hill Co., 1982, p 57.

[7] K. M. Lee, G. J. Vachtesevanos, and C. K. Kwan, Development of a spherical stepper wrist motor, J Intelligent Robotic Sys, (1988), p 225.

\section{BIOGRAPHIE}

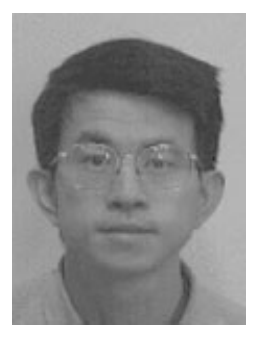

Tseng-Ti Fu is an assistant professor in the Department of Mechanical Engineering at National Taiwan University, Republic of China. His research interests are in computer-aided simulation (virtual prototyping), synthesis of mechanisms, spherical linkages, vehicle dynamics, and design evaluation methodologies. $\mathrm{He}$ received his $\mathrm{PhD}$ in engineering from the University of Cambridge, UK.
[8] C.-W. Yeh and T.-T. Fu, On the computer simulation and application in automotive steering systems by ADAMS, The 2nd Taiwan ADAMS User Conference Proceedings, Taipei, 2000.

[9] L. Chin, C. Huang, and T.-T. Fu, Dynamic simulation and validation of a mechanical system, The 3rd Taiwan ADAMS User Conference Proceedings, Taipei, 2001.

[10] C.-W. Yeh, Evaluation of steering system designs, Master dissertation, ROC: National Taiwan University, 2001.

[11] K. Fukumura, K. Haga, M. Suzuki, and K. Mori, Center-closed rotary servo valve for power steering, SAE Paper 960929, (1996).

[12] S. A. Sorby, G. Walker, M. Yano, V. Glozman, K. Kochersberger, J. Mathers, J. McKinney, J. Schulman, and M. Young, Modernization of the mechanical engineering curriculum and guidelines for computeraided engineering instruction, Comp App Eng Edu 4(7) (1999), 252-260. 